\title{
Body temperature changes before minor illness in infants
}

\author{
J A Jackson, S A Petersen, M P Wailoo
}

\begin{abstract}
This study aimed to examine the overnight temperature pattern of babies during the prodromal phase of minor illnesses. The overnight rectal temperature pattern of 123 babies was recorded weekly from about 6 to at least 16 weeks old, while parents maintained detailed records of signs of illness.
\end{abstract}

By analysis of patterns of signs and visits to the general practitioner, 86 periods of minor illness were identified, mostly upper respiratory tract infections, though it was not usually possible to identify the infection by conventional virology.

Data were analysed separately for babies who had developed an adult-like night time temperature pattern and those who had not. In both groups, obvious signs of illness were preceded by a disturbance of night time temperature pattern. Temperature was significantly raised over control weeks, though few babies were clinically febrile. The greatest temperature disturbances were seen in the three days before illness, though some disturbances were seen up to seven days before.

A similar disturbance of temperature was seen the night after diphtheria, pertussis, and tetanus immunisation, and individual responses to natural infection and immunisation were well correlated, suggesting that the temperature change is more a function of the host response than the infecting agent.

(Arch Dis Child 1994; 71: 80-83)

Infections have frequently been implicated in sudden infant death syndrome (SIDS), although overt illness before death is not commonly recognised, and postmortem examination rarely reveals signs of illness. ${ }^{1}$ This suggests that, if infections are important, infants are most vulnerable during the early incubation phase, before obvious signs have developed. During this time the host is beginning to mount humoral responses to infection which can have a variety of physiological effects. One likely change is disturbance of body temperature, as many mediators act on the hypothalamic thermoregulatory areas.

This study aimed to record patterns of rectal temperature change in babies before signs of minor illness become apparent. We have made regular overnight recordings of deep body temperature in babies at home, while parents maintained a detailed record of signs of illness, so that once illness became obvious we could analyse the preceding recordings.

Changes in overnight temperature patterns can only be interpreted in the light of developmental changes over the first few months of life. In young infants, temperature falls similarly during night and day time sleeps - to about $36 \cdot 8^{\circ} \mathrm{C}$. At an age which varies from baby to baby, temperature abruptly begins to fall further during night time sleep - to about $36 \cdot 4^{\circ} \mathrm{C}$. The age of this transition varies between 7 and 16 weeks in normal infants. ${ }^{2}$ Any changes in rectal temperature with infection, therefore, need to be related to the infant's stage of development of night time body temperature rhythms. Data have to be analysed separately for recordings made before and after the transition in night time temperature patterns.

\section{Subjects and methods}

A total of 123 subjects were recruited at or just after birth from Leicester Royal Infirmary. Many of the babies were bottle fed, had older siblings at school or nursery, and had parents who smoked, as it was thought that minor infections would be more likely in this group. Parents' consent was obtained to monitor sleeping body temperatures at weekly intervals from around 6 weeks old. Full perinatal data were collected, and parents were issued with a diary to record prospectively each day any signs of illness in their baby. A checklist of possible signs was presented to be ticked off daily. The list of signs was derived from that used in the Foundation for the Study of Infant Deaths (FSID) 'care of the next infant' (CONI) scheme, and contains 25 of the most common signs.

Once a week, from around 6 weeks, the baby was visited at home, weighed naked, and parents questioned closely on the state of the infant over the previous week. Data were also collected on the current feeding regimen, other aspects of parental care, and the health of the family. Probes were then attached to record body temperature. A soft probe, inserted $5 \mathrm{~cm}$ from the anal margin, recorded rectal temperature and a skin thermistor on the shin measured surface temperature. A third probe measured the ambient temperature of the baby's bedroom. The probes were connected to a Grant Squirrel data logger set to sample at one minute intervals throughout the night. Note was taken of items of clothing and bedding used so that a 'tog value' could be calculated according to tables produced by the Shirley Institute, Manchester. Parents were also asked to keep a detailed diary of all events 
throughout each night of recording, including nappy changes, periods of waking, and any medications given. In some infants, heart rate and oxygen saturation were recorded by a Nelcor pulse oximeter, and urine samples collected overnight and in the morning. These techniques are safe, ${ }^{3}$ and full ethical approval was obtained for the study. Only rectal temperature data will be reported in this paper.

The next day the data loggers were collected and the data downloaded to computer for analysis. They were scrutinised for evidence of lost probes or other problems. Only unblemished data were analysed further. For each night of recording, rectal temperature was abstracted at 30 minute intervals from one hour before bedtime until eight hours later.

Statistical comparisons were made by $t$ tests and two way analysis of variance.

\section{Results}

\section{SUBJECTS}

Altogether 123 babies were monitored, producing 943 recordings. On average each infant was recorded on $7 \cdot 7$ occasions. The mean (SEM) gestational age of the infants was 38.7 $(0 \cdot 29)$ weeks, with a mean birth weight of 3193 $(63.4) \mathrm{g}$. The average maternal age was $25 \cdot 8$ $(0.6)$ years. There were 64 boys and 59 girls, 20 were first children, and 69 had two or more siblings. Eleven of the infants had parents in occupational groups 1 or 2, and 48 in groups 6 or 7 . Eighty eight of the infants were bottle fed and 25 were breast fed.

\section{ILLNESS}

For every day of the study, the presence of signs of minor illness were noted by parents. The list of signs was derived from that used in the FSID CONI project. On many days, for any particular infant, at least one sign was present (for example, 'snuffles'). In order to identify episodes of minor illness for analysis, signs were scored on a simple three point scale. Signs such as sneezing, snuffles, teething, restlessness, and sweating were rated as 1 .

Signs such as reduced feeds, irritability, coughs, diarrhoea, and drowsiness were rated as 2 and visits to a general practitioner (GP) with a diagnosed infection as 3 . The total score of all the signs present was calculated for each day. A period of illness was then defined as an increase in the score by 2 or more from one recording to the next. By this definition, 86 periods of minor illness were identified. The mean (SEM) increase in score was $3.60(0.31)$. The majority of babies were seen by a GP or health visitor and a diagnosis of illness made; $59 \%$ were classed as upper respiratory tract infections.

For the majority of babies, temperature data were available before, during, and after the illness. In some cases, however, data were not available for all three weeks due to logging problems, refusal by the parents, or because the infant had developed a mature temperature pattern on recovery from the illness and had therefore changed over a 'postrhythm' category.

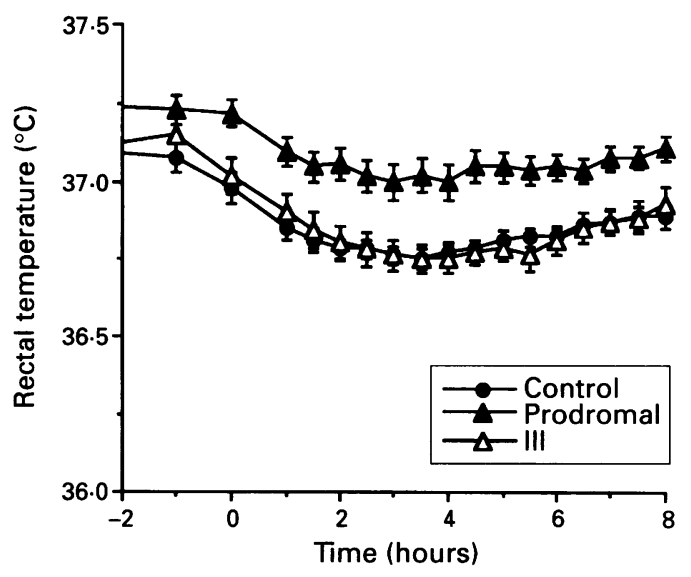

Figure 1 The overnight rectal temperature patterns of 33 prerhythm control babies, 51 prerhythm prodromal babies
(that is before illness), and 43 prerhythm ill babies. Time 0 (that is before illness), and 43 prerhythm ill babies. Time
indicates bedtime. Each point shows the mean (SEM).

\section{BODY TEMPERATURE CHANGES}

Once a period of illness was identified, the preceding overnight temperature recording was abstracted, together with the following recording. The mean (SEM) interval between the preceding recording and the first day of illness was $4.6(1.6)$ days. The interval between the first day of illness and the following temperature recording averaged $2 \cdot 2(0 \cdot 4)$ days.

Figure 1 compares the body temperature patterns of the infants, in the 'prerhythm' stage of development, with the recording before illness ('prodromal'), the recording made once illness was known ('ill'), and a recording made before or after when the baby was apparently neither ill nor incubating illness ('control'). Sleeping body temperature was significantly higher in the prodromal phase than when the baby was well $(\mathrm{F}=146.37$ with $1,1076 \mathrm{df}$, $\mathrm{p}<0.001$, two way analysis of variance). Surprisingly, none of the parents reported giving paracetamol or other antipyretic drugs once the baby was known to be ill.

Figure 2 shows comparable data for babies who had developed an adult-like night time body temperature pattern (postrhythm), defined by a rectal temperature falling below $36.5^{\circ} \mathrm{C}$ for at least one hour during the night, for two successive weekly recordings. Again,

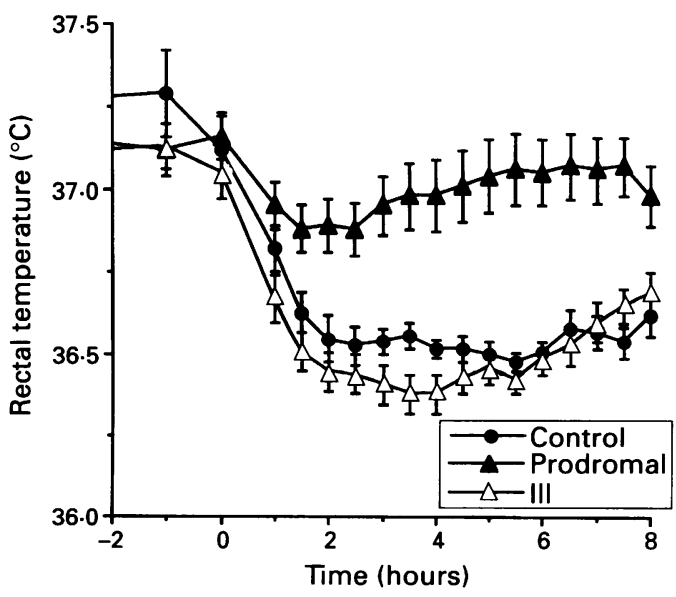

Figure 2 The overnight rectal temperature patterns of postrhythm control babies, postrhythm prodromal babies (that is before illness), and postrhythm ill babies. Time 0 indicates bedtime. Each point shows the mean (SEM). 


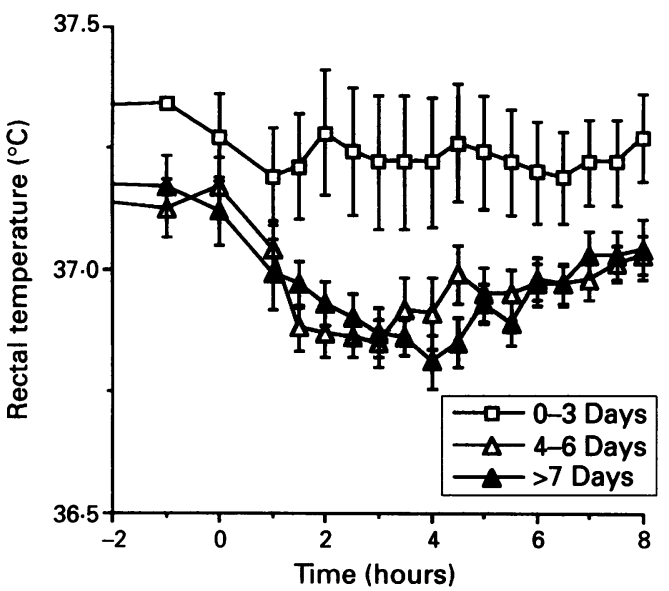

Figure 3 The overnight rectal temperature patterns of prerhythm babies at different intervals before obvious signs of illness appeared. Nineteen babies were monitored up to three days, 15 babies at four to six days, and 14 babies more than seven days before illness. Time 0 indicates bedtime. Each point shows the mean (SEM).

body temperature was significantly raised during the prodromal phase $(F=101.85$ with $1674 \mathrm{df}, \mathrm{p}<0.001$, two way analysis of variance).

\section{TIME COURSE OF BODY TEMPERATURE}

\section{CHANGES}

By chance, recordings before illness were made at different intervals before obvious signs appeared. Figure 3 compares the prodromal body temperature changes recorded in babies seven or more days, four to six days, and three days or less before illness. The greatest changes in temperature were seen in the three days before illness $(\mathrm{F}=109.35$ with $1368 \mathrm{df}$, $\mathrm{p}<0.001$, two way analysis of variance), although there was a significant difference up to seven days before ( $F=15 \cdot 77$ with $1296 \mathrm{df}$, $\mathrm{p}<0.001$, two way analysis of variance).

SPECIFICITY OF BODY TEMPERATURE CHANGES Figure 4 shows the distribution of the maximum body temperature attained between two and five hours after bedtime by prodromal

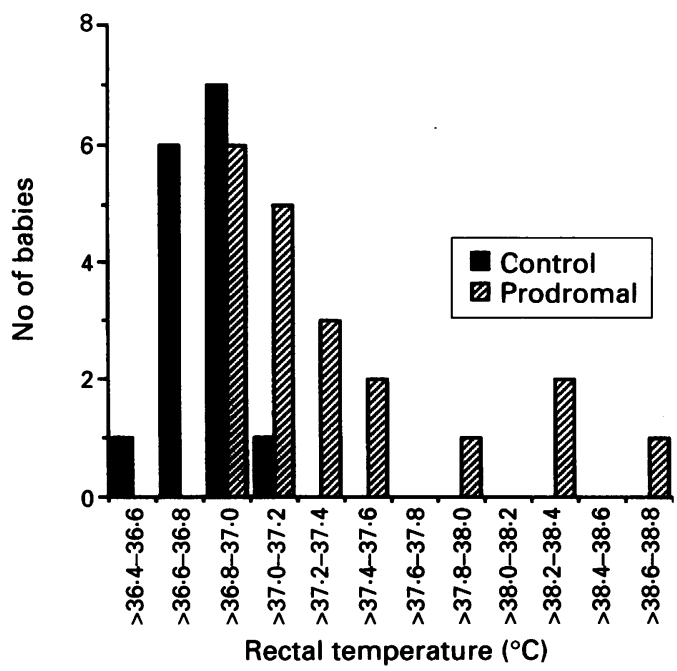

Figure 4 The maximum rectal temperature attained two to five hours after bedtime in prerhythm infants up to three days before illness.

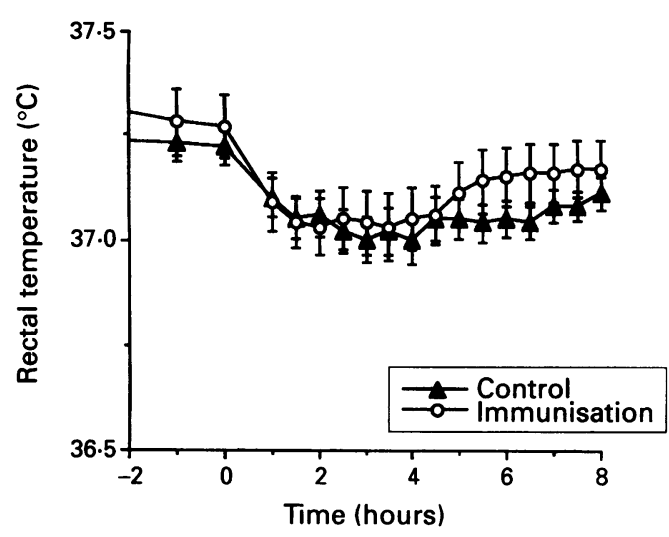

Figure 5 The overnight rectal temperature patterns of 16 prerhythm babies during the prodromal phase of illness and of the same 16 babies the night after immunisation against DPT and polio. Time 0 indicates bedtime. Each point shows the mean (SEM).

and control prerhythm babies three days before illness. Only $6 \%(5 / 86)$ of the infants were clinically febrile $\left(>38^{\circ} \mathrm{C}\right)$. Taking as a criterion rectal temperature above $37^{\circ} \mathrm{C}$ for at least one hour during the night, $65 \%$ of illnesses were preceded by a body temperature disturbance but such a temperature disturbance was only observed on $21.2 \%$ of nights when no illness subsequently occurred after three days.

Taking the same criterion as for prerhythm infants, $86 \%$ of illness occurring in postrhythm infants was preceded by a body temperature disturbance but this type of temperature disturbance was only observed on $10.5 \%$ of nights when no illness subsequently occurred after three days.

\section{RELATIONSHIP OF BODY TEMPERATURE}

CHANGES TO THOSE AFTER IMMUNISATION

The body temperature pattern of 62 of the babies was also monitored the night after at least one of their immunisations against diphtheria, pertussis, and tetanus (DPT) and polio. Immunisation against Haemophilus influenzae type $\mathrm{b}$ was given to only four infants. Figure 5 compares the body temperature pattern of prerhythm infants in the prodromal phase of illness and after immunisation. In both cases body temperature was significantly raised $(F=86.16$ with $1725 \mathrm{df}, \mathrm{p}<0.001$, two way analysis of variance for after immunisation). There was no significant difference between the prodromal pattern and that after immunisation.

In many cases, DPT immunisation was delayed by minor illness until after an adultlike temperature rhythm had developed, and so the prodromal responses and immunisation effects could not be observed in the same babies at the same stage of development. In 16 cases, however, both prodromal recordings and recordings after immunisation were available in prerhythm infants. There was a remarkable association between the maximum temperatures attained between two and five hours into the night after immunisation and during the prodromal phase $(r=0.891$ $\mathrm{p}<0.001$, product moment correlation). 


\section{Discussion}

This study depended upon 'catching' a variety of minor illnesses in babies before obvious signs had developed. It is obviously, therefore, fraught with all the difficulties of naturalistic observations. Even so, a reasonable number of illnesses were identified. The nature of illness varied from infant to infant, though the vast majority were upper respiratory infections. Presumably the infecting agent also varied, though as nasopharyngeal swabs taken from the infants during the prodromal phase invariably proved negative by conventional virology and bacteriology, we could rarely identify them. Three of the infants who went on to be ill enough to warrant hospitalisation were eventually identified as infected with respiratory syncytial virus. The severity of illness also varied from barely enough to cause concern to almost life threatening.

We chose to identify illness by parental reporting of signs. Clearly, some parents will report more readily than others, and it is impossible to ensure that signs such as snuffles are interpreted in the same way by all observers. In most cases of illness, however, the parents were sufficiently concerned to seek the advice of a GP, and a clinical diagnosis of illness was made.

Without deliberately infecting infants, however, it is difficult to see how these problems could be overcome, and what is remarkable is the consistency of the temperature pattern observed in the period before illness appears. A disturbance of sleeping body temperature preceded obvious signs of illness by several days. During this period parents often reported vague feelings that their infants were 'not right', and many babies were reported as drowsy, or feeding less well than usual. Indeed weight gain in the week preceding illness was significantly reduced.

This prodromal period could be a period of vulnerability. We made a few measurements of urine-free cortisol that showed an increase typical of metabolic stress. Heart rate was also raised. We will report elsewhere one case of a baby who died of SIDS during monitoring in whom sleeping temperature was identical to the prodromal pattern we describe here.

If these illnesses were due to infection, which seems likely, then the data suggest a prolonged period during which the infection is becoming established, and the host is mounting a response, which includes body temperature changes. A previous author has suggested that such a time lapse does exist. ${ }^{4}$ These temperature changes may be produced by circulating substances such as cytokines. It is likely that such a host response is also mounted after immunisation, and the similarity between response in the prodromal phase of illness and after DPT immunisation suggests that the extent of the temperature change (and therefore presumably the humoral changes producing it) is more a property of the host than the infecting agent. This may mean that some infants will mount a more vigorous host response than others to the same challenge, which may affect their vulnerability to sudden death during this phase of illness.

In conclusion, we have demonstrated that minor illnesses of infancy are preceded by a period in which temperature patterns are disturbed in a characteristic way, presumably indicating a host response to an infecting agent. The monitoring of this temperature pattern offers an opportunity to study infants in this vital stage when they are ill, but nobody is really aware of it, and they may be particularly vulnerable.

We thank the Foundation for the Study of Sudden Infant Deaths and Babes in Arms for their support.

1 Fleming KA. Viral respiratory infection and SIDS. $\mathcal{f}$ Clin Pathol 1992; 45 (suppl): 29-32.

2 Lodemore M, Petersen SA, Wailoo MP. Development of night time rhythms over the first six months of life. Arch Dis Child 1991; 66: 521-4.

3 Wailoo MP, Petersen SA, Whittaker H, Goodenough P Sleeping body temperature in 3-4 month old infants. Arch Dis Child 1989; 64: 596-9.

4 Roden AT. Clinical assessment of the common cold. Proc $R$ Soc Med 1958; 51: 271-3. 